\title{
Fiber Integrated Wavelength Converter Based on a Silicon Core Fiber with a Nano-spike Coupler
}

\author{
Meng Huang, Haonan Ren, Ozan Aktas, Li Shen, Jian Wang, Thomas W. Hawkins, \\ John Ballato, Ursula J. Gibson, and Anna C. Peacock
}

\begin{abstract}
An all-fiber integrated nonlinear silicon photonic wavelength converter has been proposed and fabricated using the silicon core fiber platform. The silicon fiber was spliced directly to a conventional single mode fiber, facilitated via an inverse tapered nano-spike that helped to reduce the mode mismatch between the different core materials. Four-wave mixing-based wavelength conversion with an efficiency as high as $-22.1 \mathrm{~dB}$ has been achieved for selected wavelengths across the $\mathrm{C}$-band in a device length of only $\sim 1 \mathrm{~cm}$. Successful conversion of quadrature phase-shift keying signals at a 20-Gb/s bitrate, with a 1 to $2 \mathrm{~dB}$ penalty level at the bit error ratio $(B E R)=3.8 \times 10^{-3}$, was used to demonstrate the suitability of the silicon fiber device for the construction of ultra-compact, all-fiber-based optical signal processing systems.
\end{abstract}

Index Terms-Silicon photonics, wavelength converters, fourwave mixing, nonlinear fiber optics, fiber design and fabrication, fiber materials.

\section{INTRODUCTION}

Nonlinear silicon photonics has been widely investigated using integrated silicon waveguide platforms, with the aim of developing compact devices for future telecommunication networks. Within this field, components that exploit fourwave mixing (FWM) to generate new frequencies for alloptical signal processing applications have attracted significant research interest [1], [2]. Owing to the intrinsic high nonlinearity and tight mode confinement within silicon waveguides, they offer several advantages over more conventional fiber-based components such as small device footprints and

Manuscript received XX XX, 2019; revised XX XX, 2019; accepted XX XX, 2019. Date of publication XX XX, 2019; date of current version XX XX, 2019. This work was supported by the following research funds: National Natural Science Foundation of China (NSFC) (61705072,61761130082); Engineering and Physical Sciences Research Council (EPSRC) (EP/P000940/1, EP/N013247/1); Royal Society-Newton Advanced Fellowship; the Norwegian Research Council (262232); the J. E. Sirrine Foundation. (Corresponding author: Li Shen)

M. Huang, L. Shen, and J. Wang are with Wuhan National Laboratory for Optoelectronics, School of Optical and Electronic Information, Huazhong University of Science and Technology, Wuhan 430074, Hubei, China. Li Shen is also at Optoelectronics Research Centre, University of Southampton, Southampton, SO17 1BJ, United Kingdom (e-mail: lishen@hust.edu.cn).

H. Ren, O. Aktas, and A. C. Peacock are with Optoelectronics Research Centre, University of Southampton, Southampton, SO17 1BJ, United Kingdom.

T. W. Hawkins, and J. Ballato are with Center for Optical Materials Science and Engineering Technologies and Department of Materials Science and Engineering, Clemson University, Clemson, SC 29634, USA.

U. J. Gibson is with Department of Physics and Porelabs, Norwegian University of Science and Technology, N-7491 Trondheim, Norway. She is also at Department of Applied Physics, KTH Royal Institute of Technology, Stockholm 10044, Sweden.

The data for this work is accessible through the University of Southampton Institutional Research Repository (DOI:10.5258/SOTON/D1038). lower power thresholds [3], [4]. Notable FWM-based silicon device demonstrations that have been conducted within the telecommunications band include wavelength converters [1], [5], demultiplexers [6], optical parametric amplifiers [7], alloptical signal regenerators [8], and optical isolators [9]. To date, most of these nonlinear demonstrations have employed free space systems to couple the pump light into the silicon waveguides. However, before these systems can be deployed in practical telecommunication systems, robust, alignment-free components are required.

Silicon core fibers (SCFs) are emerging as an alternative nonlinear platform that combines the benefits of the fiber geometry with the advantages of the highly nonlinear silicon material systems [10]. These fibers can be fabricated using conventional drawing tower procedures, but the core sizes produced via this method are typically on the order of $\sim 10 \mu \mathrm{m}$ [11]. To obtain the few micrometer sized cores required for nonlinear applications, a post-draw tapering procedure has been recently developed [12]. Similar to the planar silicon waveguides, these small core SCFs have shown great potential for nonlinear applications [13]. More recently, the tapering process has been extended to produce nano-spike couplers at the input facet of the SCFs, enabling robust fusion splicing to conventional single mode fibers (SMFs), while retaining efficient optical coupling into the silicon core [14]. This work has opened a route for the SCFs to find use in practical, allfiber integrated nonlinear photonic systems.

In this letter, we fabricated a SCF-based nonlinear wavelength converter (NWC) with a SMF pigtail input coupler and demonstrated its use for data conversion. The SCFNWC has been designed to facilitate FWM-based conversion within the telecom band, with conversion efficiencies as high as $-22.1 \mathrm{~dB}$. Wavelength conversion of quadrature phaseshift keying (QPSK) signals with a $20-\mathrm{Gb} / \mathrm{s}$ bitrate has been achieved using a SCF that is only about a centimeter long. BER measurements reveal that the conversion of the modulated signals takes place with a negligible optical signal-tonoise ratio $(\mathrm{OSNR})$ penalty (at the BER $=3.8 \times 10^{-3}$ ). We anticipate that with continued efforts to reduce the coupling and transmission losses in these SCF-NWC devices, they will unlock new possibilities for all-fiber signal processing applications in telecommunications systems.

\section{FABRICATION OF AN ALL-FIBER INTEGRATED WAVELENGTH CONVERTER}

The proposed all-fiber integrated SCF-NWC system is shown in Fig. 1(a). The primary element of the NCW is a 

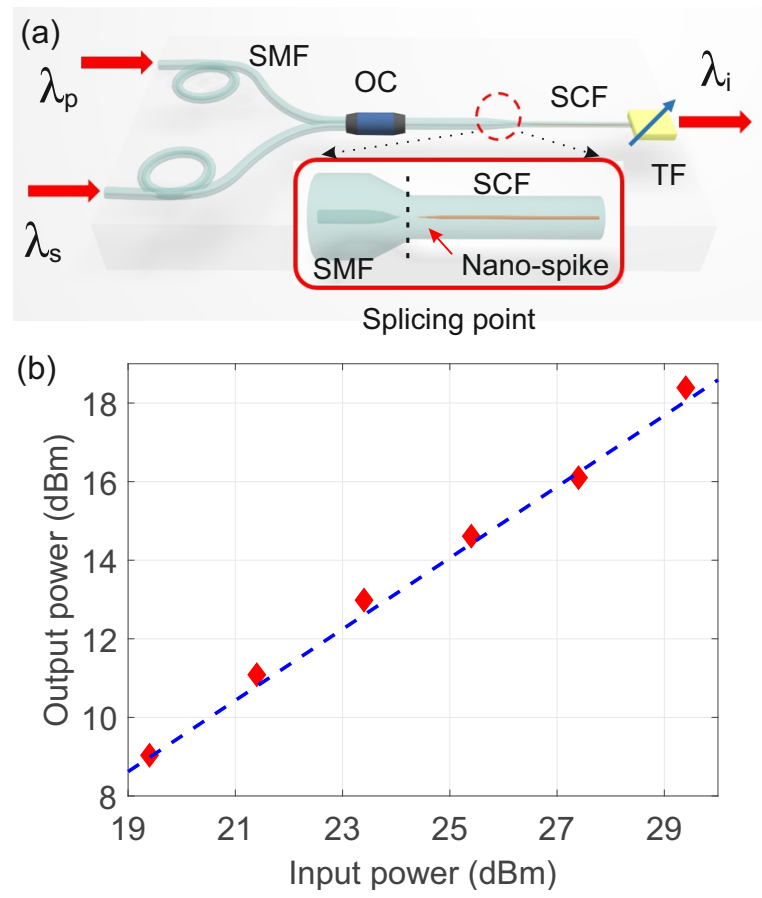

Fig. 1. (a) Schematic of the proposed FWM-based all-fiber system. OC: optical coupler. TF: tunable filter. Inset: SCF-NWC design showing the SCF spliced to a tapered SMF via a nano-spike coupler. (b) Transmission properties of the SCF-NCW as a function of input power.

centimeter long SCF, as shown in the inset, where the highly nonlinear core is exploited for efficient wavelength conversion. The converter is based on a simple degenerate FWM process, in which two photons from the high power pump wave-mix and generate a single signal photon and a converted idler photon at their respective wavelengths. Finally the converted idler is selected at the output by an external filter. All the optical components are fiber-based, thus the proposed SCFNWC system has the potential to be very robust.

The converter was fabricated from SCFs that were produced via the molten core drawing (MCD) method, as detailed in Refs. [11], [15]. Using this method, tens of meters of SCFs were drawn with silicon core/silica cladding diameters of $\sim 5.5 \mu \mathrm{m} / 144 \mu \mathrm{m}$. A two step post-draw tapering procedure was then used to produce the SCF-NWC device. Firstly, the SCF was tapered to scale down the dimensions to obtain core/cladding diameters of $\sim 1.1 \mu \mathrm{m} / 30 \mu \mathrm{m}$ over a $1.5 \mathrm{~cm}$ length, and to improve the crystallinity of the as-drawn polycrystalline core [16]. A second step was then used to form the nano-spike at the end of the SCF by adjusting the tapering parameters, as detailed in Ref. [14]. The section of the SCF attached to the nano-spike can then be cleaved and spliced to a SMF that has also been tapered to have a matching outer diameter, as illustrated in the inset of Fig. 1(a). The final fabricated SCF-NWC consists of a $1.1 \mathrm{~cm}$ long SCF with a $\sim 200 \mu \mathrm{m}$ long nano-spike, connected to a SMF pigtail with a $4 \mathrm{~mm}$ long tapered transition region to facilitate mode coupling between the two structures. The input of the fabricated device was then directly connected to a fiber laser source for the following experiments.
Linear optical characterization of the nano-spike integrated SCF structures has previously been investigated in Ref. [14]. The linear propagation loss of the SCF with a $1.1 \mu \mathrm{m}$ core used in the SCF-NWC device was measured to be $2 \mathrm{~dB} / \mathrm{cm}$ at $1550 \mathrm{~nm}$ via the cut-back method. The optical transmission measurement of the fabricated SCF-NWC device was undertaken using the same experimental setup, as detailed in Ref. [14]. The total insertion loss was calculated to be $\sim 11 \mathrm{~dB}$ from Fig. 1(b), including losses at the output facet composed of a $1.5 \mathrm{~dB}$ reflection and $1 \mathrm{~dB}$ mode mismatch (estimated using Lumerical mode solution software). Thus, the coupling efficiency into the SCF section of the NCW was estimated to be $\sim 6.5 \mathrm{~dB}$, which included contributions from the splice loss $\sim 2 \mathrm{~dB}$ and the nano-spike coupling loss $\sim 4.5 \mathrm{~dB}$. The nanospike coupling loss could be reduced to $<1 \mathrm{~dB}$ by decreasing the silica cladding diameters at the join to $\sim 10 \mu \mathrm{m}$. This level of input coupling loss would be competitive with the best coupling losses reported for planar silicon waveguides that make use of a similar inverse taper design, but with the benefits of reduced alignment complexity and a more robust connection to the input fiber. Although this current SCF-NWC device employs free space output coupling, it could eventually be fully integrated by fabricating nano-spikes on both ends of the SCF. However, we are currently restricted from making such a structure due to the lack of a suitable holder to fix the micrometer diameter fiber in the taper rig for the formation of the second spike.

\section{OPTICAL PROPERTIES OF THE WAVELENGTH CONVERTER}

In order to evaluate the nonlinear optical performance of the fabricated SCF-NWC device, degenerate FWM experiments were performed via the experimental setup from Fig. 1(a) (with the TF replaced by an OSA to resolve the spectral components), pumped by a CW laser source in the telecom band. The $\mathrm{CW}$ pump wave and a lower power tunable $\mathrm{CW}$ signal wave were combined in an optical coupler before launching into the SCF-NWC device. Fig. 2(a) shows a typical spectrum recorded at the device output, measured by an optical spectrum analyzer (OSA - Yokogawa-AQ6370C). Owing to the relatively short device length, the walk-off between the pump and signal beams can be neglected and will not reduce the conversion efficiency (defined as $P_{\text {idler }} / P_{\text {signal }}$ ) at the device output. The measured FWM conversion efficiency as a function of pumpsignal detuning is shown in Fig. 2(b). In the experiment, the pump wavelength is fixed at $\lambda_{p}=1550.1 \mathrm{~nm}$ and the idler wavelength can be tuned from $1550.4 \mathrm{~nm}$ to $1557 \mathrm{~nm}$ as the signal wavelength is varied. The conversion efficiency decreases as the signal wavelength moves away from the pump wavelength and the tunable range is $\sim 7 \mathrm{~nm}$. This limited bandwidth is attributed to the large normal dispersion of the section of the SCF-NWC device with a micron-sized core, which is estimated to be $\beta_{2} \sim 0.4 \mathrm{ps}^{2} \mathrm{~m}^{-1}$ from finite-element modelling of the modal properties [17]. Further investigations to accurately map the dispersion profile of the full SCFNWC device, which would allow for a quantitative comparison with the experimental data, are beyond the scope of this 

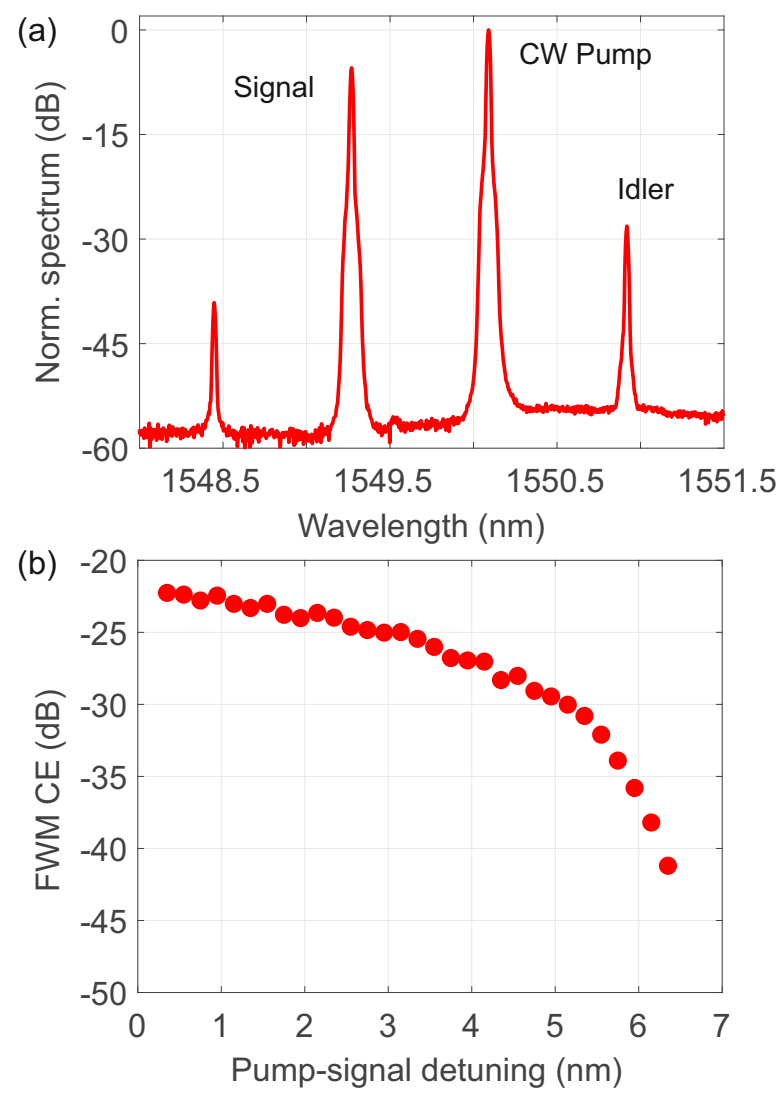

Fig. 2. (a) FWM spectrum recorded at the device output. (b) FWM efficiency as a function of pump-signal detuning.

work [18]. The maximum measured conversion efficiency is $\sim-22.1 \mathrm{~dB}$ with a coupled input pump power of $22.9 \mathrm{dBm}$, which is a comparable efficiency to what has been obtained in planar silicon nanowires [19]. However, to better compare these results, a normalized efficiency is defined that takes into account the device length and coupled pump power [20]:

$$
\eta_{\text {norm }}=\frac{100 \times\left(P_{\text {idler }} / P_{\text {signal }}\right)}{P_{\text {pump }}^{2} L^{2}}
$$

Using this definition, a threefold improvement in the figure of merit for the SCF-NWC ( $\eta_{\text {norm }}=16.2 \% \mathrm{~W}^{-2} \mathrm{~mm}^{-2}$ ) has been obtained compared to the planar silicon nanowire ( $\eta_{\text {norm }}=5.29 \% \mathrm{~W}^{-2} \mathrm{~mm}^{-2}$ in Ref. [19]). The conversion efficiency in the SCF-NWC could be further improved by increasing the device length and/or peak pump power, i.e., by using a pulsed pump. Fig. 1(b) clearly shows that the measured output powers increase linearly for input powers up to $29.4 \mathrm{dBm}$, indicating that there is no observable nonlinear absorption in our experiment for this modest pump level. Moreover, there was no observable degradation at the splicing point between the SCF and the SMF over many months of use, indicating that this SCF-NWC device could provide robust and stable operation for nonlinear applications requiring similar pump power levels.

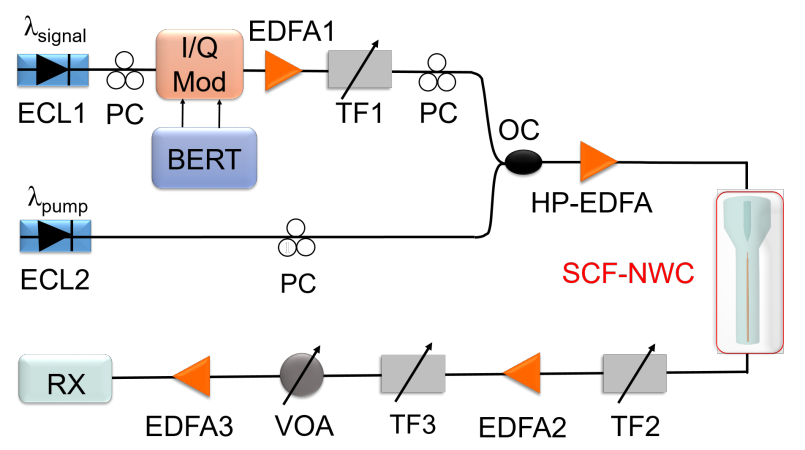

Fig. 3. Schematic of wavelength conversion setup. ECL: external cavity laser; BERT: bit error rate tester; TF: tunable filter; OC: optical coupler; PC: polarization controller; VOA: variable optical attenuator; RX: receiver.

\section{All FibER WAVELENGTH CONVERSIONS}

Fig. 3 shows the experimental set up for FWM-based wavelength conversion of widely used QPSK signals. Two tunable external cavity lasers (ECL1 and ECL2) were used as the pump and signal sources. The output light of ECL1 was modulated with QPSK data signals from a single-polarization optical I/Q modulator. The electric signals were generated at a symbol rate of 10-Gbaud using a two-channel BER tester (Alnair Labs SeBERT-100E) and then amplified to between 4 to $5 \mathrm{~V}$ to drive the I/Q modulator. The I/Q modulator was operated in a single-sideband-carrier suppressed (SSBCS) mode under a biasing voltage of $7 \mathrm{~V}$. The modulated 10-Gbaud QPSK signal was then amplified by an erbiumdoped optical fiber amplifier (EDFA1), followed by a bandpass filter (TF1) with a bandwidth of $0.8 \mathrm{~nm}$ to suppress the amplified spontaneous emission (ASE) noise. Two polarization controllers (PCs) were used to align the polarization states of the pump and signal waves to achieve the optimum conversion efficiency. The pump wave and the modulated signal were combined by a $3 \mathrm{~dB}$ optical coupler, amplified using a highpower EDFA (HP-EDFA), and launched into the SCF-NWC. Unlike planar silicon waveguides, no additional polarization control is needed in the setup because the SCF is polarization insensitive, owing to its cylindrical symmetry. At the output, the converted idler was selected using a second filter (TF2) and sent to a pre-amplifier (EDFA2), due to the relatively low power level of the converted idler. A third filter (TF3) was used to suppress the ASE noise originating from EDFA2. The bandwidths for TF2 and TF3 are also $0.8 \mathrm{~nm}$. A variable optical attenuator (VOA) and a low noise EDFA (EDFA3) were employed to adjust the received OSNR for the BER measurements.

In the nonlinear conversion experiments, various converted idler wavelengths can be generated simply by tuning the pump and signal wavelengths. Fig. 4(a) shows a typical FWM spectrum obtained at the output of the SCF-NWC for a modulated signal at $1554.6 \mathrm{~nm}$. The measured QPSK constellations of the input signal and idler waves are shown in the insets of Fig. 4(a), clearly indicating the modulated data has been successfully converted. The background noise on the weakly converted idler mainly causes performance deterioration. BER 

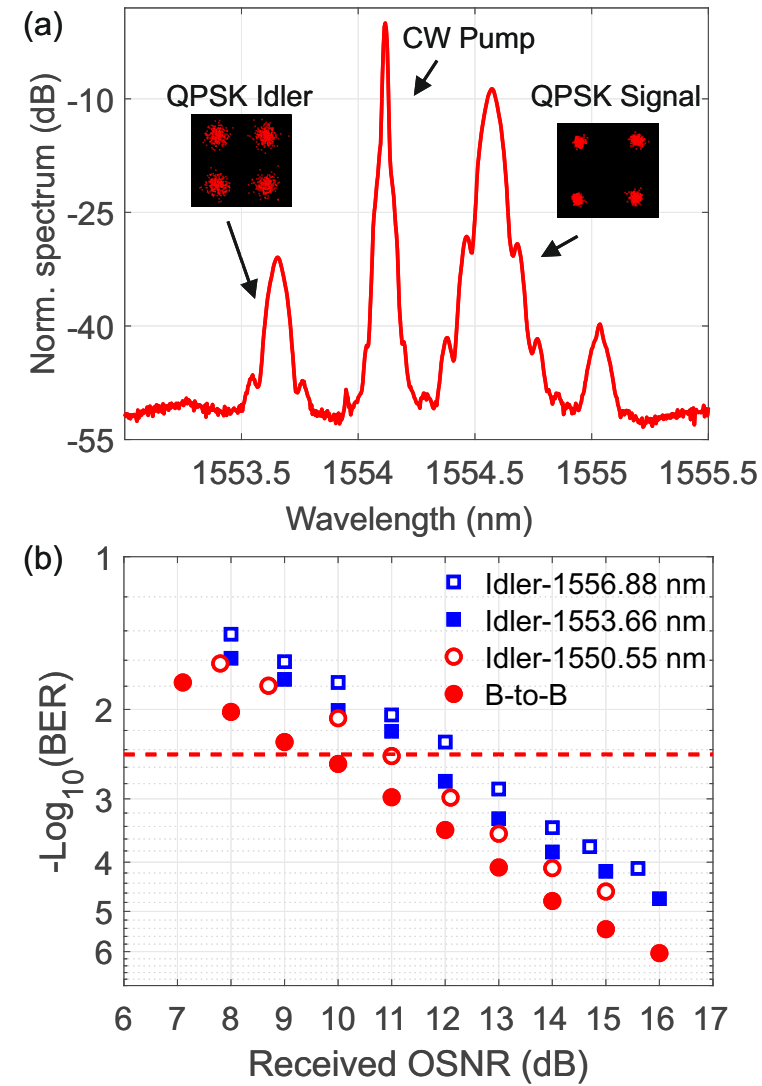

Fig. 4. (a) QPSK spectrum recorded at the waveguide output. Signal and idler constellation diagrams are shown as insets. (b) BER measurements for $20 \mathrm{~Gb} / \mathrm{s}$ QPSK signals at different idler and B-to-B signal wavelengths.

performance was subsequently evaluated across the C-band as a function of the received OSNR for the back-to-back (B-to-B) signal and various converted idler wavelengths, as labeled in Fig. 4(b). In order to obtain sufficient OSNR of the generated idlers for coherent detection, the pump-signal detuning was kept to $\sim 0.35 \mathrm{~nm}$, to allow maximum conversion efficiencies for these three idlers. The coupled input power of the SCFNWC device was fixed at $22.9 \mathrm{dBm}$. The observed OSNR penalty is around $1 \mathrm{~dB}$ at a BER of $3.8 \times 10^{-3}(7 \%$ forward error correction threshold) for QPSK wavelength conversion with an idler at $1550.55 \mathrm{~nm}$. This increases slightly to $\sim 2 \mathrm{~dB}$ for the $1553.66 \mathrm{~nm}$ and $1556.88 \mathrm{~nm}$ idlers, mainly due to the variation in the conversion efficiency. Note, this BER level was chosen as a hard decision forward error correction threshold and used to evaluate the performance of the device for use in telecom systems [21]. Thus, these results reveal that successful high speed wavelength conversion of QPSK signals can be obtained in our SCF-NWC, indicating their potential for use in practical telecom applications.

\section{CONCLuSion}

To conclude, a FWM-based conversion of $20 \mathrm{~Gb} / \mathrm{s}$ QPSK signals in an all-fiber integrated SCF-NWC device with a length of only $\sim 1 \mathrm{~cm}$ was demonstrated. The SCF was robustly spliced to $\mathrm{SMF}$, facilitated via a nano-spike tapered coupler, so that it could be directly connected to fiber laser sources, offering a considerably higher power damage threshold than planar-based devices. Wavelength conversion was demonstrated using a CW pump source, achieving a peak conversion efficiency of $-22.1 \mathrm{~dB}$ with a modest pump power of $22.9 \mathrm{dBm}$. Constellation diagrams and BER testing were used to evaluate the conversion of high speed complex QPSK modulated signals, returning a 1 to $2 \mathrm{~dB}$ power-penalty at the $\mathrm{BER}=3.8 \times 10^{-3}$. These results highlight the suitability of the SCF platform for integration in existing fiber networks and its potential for use in nonlinear all-optical signal processing applications for future communication systems.

\section{REFERENCES}

[1] R. L. Espinola, J. I. Dadap, R. M. Osgood, S. J. McNab, and Y. A. Vlasov, "Four-wave mixing in silicon wire waveguides," Opt. Express, vol. 13, no. 11, pp. 4341-4349, May 2005.

[2] K. Li and A. C. Foster, "Parametric nonlinear silicon-based photonics," Proceedings of the IEEE, vol. 106, no. 12, pp. 2196-2208, Dec. 2018.

[3] M. Hirano, T. Nakanishi, T. Okuno, and M. Onishi, "Silica-based highly nonlinear fibers and their application," IEEE J. Sel. Top. Quantum Electron., vol. 15, no. 1, pp. 103-113, Jan. 2009.

[4] J. Leuthold, C. Koos, and W. Freude, "Nonlinear silicon photonics," Nature Photon., vol. 4, pp. 535-544, Jul. 2010.

[5] H. Rong, Y. Kuo, A. Liu, M. Paniccia, and O. Cohen, "High efficiency wavelength conversion of $10 \mathrm{~Gb} / \mathrm{s}$ data in silicon waveguides," Opt. Express, vol. 14, no. 3, pp. 1182-1188, Feb. 2006.

[6] C. Koos et al., "All-optical high-speed signal processing with silicon-organic hybrid slot waveguides," Nature Photon., vol. 3, pp. 216219, Apr. 2009.

[7] M. A. Foster et al., "Broad-band optical parametric gain on a silicon photonic chip," Nature, vol. 441, pp. 960-963, Jan. 2006.

[8] R. Salem et al., "Signal regeneration using low-power four-wave mixing on silicon chip," Nature Photon., vol. 2, pp. 35-38, Jan. 2008.

[9] K. Wang et al., "Four-wave-mixing-based silicon integrated optical isolator with dynamic non-reciprocity," IEEE Photon. Technol. Lett., vol. 28, no. 16, pp. 1739-1742, Aug. 2016.

[10] A. C. Peacock, U. J. Gibson, and J. Ballato, "Silicon optical fibres-Past, present, and future," Adv. Phys.: X, vol. 1, no. 1, pp. 114-127, Mar. 2016.

[11] J. Ballato and A. C. Peacock, "Perspective: Molten core optical fiber fabrication-A route to new materials and applications," APL Photonics, vol. 3, no. 12, pp. 120903, Nov. 2018.

[12] F. H. Suhailin et al., "Tapered polysilicon core fibers for nonlinear photonics," Opt. Lett., vol. 41, no. 7, pp. 1360-1363, Apr. 2016.

[13] H. Ren et al., "Nonlinear optical properties of polycrystalline silicon core fibers from telecom wavelengths into the mid-infrared spectral region," Opt. Mater. Express, vol. 9, no. 3, pp. 1271-1279, Feb. 2019.

[14] H. Ren et al., "Tapered silicon core fibers with nano-spikes for optical coupling via spliced silica fibers," Opt. Express, vol. 25, no. 20, pp. 24157-24163, Oct. 2017.

[15] E. F. Nordstrand, A. N. Dibbs, A. J. Eråker, and U. J. Gibson, "Alkaline oxide interface modifiers for silicon fiber production," Opt. Mater. Express, vol 3, no. 5, pp. 651-657, Apr. 2013.

[16] Y. Franz et al., "Material properties of tapered crystalline silicon core fibers," Opt. Mater. Express, vol. 7, no. 6, pp. 2055-2061, Jun. 2017.

[17] Q. Lin et al., "Ultrabroadband parametric generation and wavelength conversion in silicon waveguides," Opt. Express, vol. 14, no. 11, pp. 4786-4799, May 2006.

[18] S. Mas, J. Matres, J. Marti, and C. J. Oton, "Accurate Chromatic Dispersion Characterization of Photonic Integrated Circuits," IEEE Photon. J., vol. 4, no. 3, pp. 825-831, Jun. 15, 2012.

[19] R. Adams et al., "Wavelength conversion of 28 GBaud 16-QAM signals based on four-wave mixing in a silicon nanowire," Opt. Express, vol. 22, no. 4, pp. 4083-4090, Feb. 2014.

[20] A. Trita et al., "Ultra-high four wave mixing efficiency in slot waveguides with silicon nanocrystals," Appl. Phys. Lett., vol. 99, no. 19, pp. 191105, Oct. 2011.

[21] F. D. Ros et al., "Wavelength conversion of QAM signals in a low loss CMOS compatible spiral waveguide," APL Photonics, vol. 2, no. 4, pp. 046105, Mar. 2017. 